Original Article (short paper)

\title{
Acute effects of static versus proprioceptive neuromuscular facilitation stretching volume on the maximum number of repetitions and perceived exertion
}

\author{
Flavio J. Mangueira ${ }^{1}$, Maria S. Cirilo-Sousa ${ }^{2,3,4}$, Jefferson S. Novaes ${ }^{5,6}$, Pablo B. Costa ${ }^{7}$, Thais Rodrigues-Rodrigues ${ }^{2}$, \\ Gabriel R. Neto ${ }^{2,3,8,9}$ \\ ${ }^{1}$ Faculdade de Filosofia Ciências e Letras de Cajazeiras, FAFIC, Coordination of Physical Education, Cajazeiras, PB, Brazil, ${ }^{2}$ Uni- \\ versidade Federal da Paraiba, UFPB, Department of Physical Education, Kinanthropometry and Human Development Laboratory, \\ João Pessoa, PB, Brazil; ${ }^{3}$ Universidade Federal da Paraíba, UFPB, Department of Physical Education, Programa Associado de \\ Pós-graduação em Educação Física, João Pessoa, PB, Brazil; ${ }^{4}$ Universidade Regional do Cariri, URCA, Department of Physical

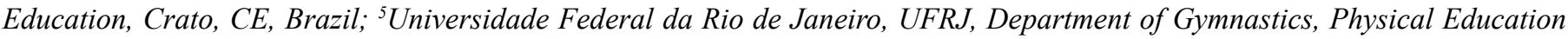 \\ Graduate Program, Rio de Janeiro, RJ, Brazil, ' ${ }^{6}$ niversidade Federal de Juiz de Fora, UFJF, Department of Physical Education, \\ Physical Education Post Graduation Program, Juiz de Fora, MG, Brazil; ' ${ }^{\prime}$ niversity of California, Department of Kinesiology, \\ Fullerton, Exercise Physiology Laboratory, CA, USA, ${ }^{8}$ Faculdade de Enfermagem e Medicina Nova Esperança, FAMENE/FACENE, \\ Coordination of Physical Education, Professional Master's in Family Health, João Pessoa, PB, Brazil, ${ }^{9}$ Centro de Ensino Superior \\ e Desenvolvimento, CESED, UNIFACISA/FCM/ESAC, Coordination of Physical Education, Campina Grande, PB, Brazil
}

\begin{abstract}
Aims: The purpose of this study was to examine the acute effects between different volumes of static stretching (SS) and proprioceptive neuromuscular facilitation (PNF) stretching (30 and 60 seconds) on the maximum number of repetitions at 40 and $80 \% 1 \mathrm{RM}$ and rating of perceived exertion (RPE). Methods: Eleven recreationallytrained women $(26.45 \pm 5.27$ years $)$ performed ten experimental protocols using a repeated measures crossover design: (a) $30 \mathrm{~s}$ of SS followed by $40 \%$ of 1RM (SS1); (b) $60 \mathrm{~s}$ of SS followed by 40\% of 1RM (SS2); (c) $30 \mathrm{~s}$ of SS followed by $80 \%$ of 1 RM (SS3); (d) 60 s of SS followed by $80 \%$ of 1RM (SS4); (e) 30 s of PNF followed by 40\% of 1RM (PNF1); (f) $60 \mathrm{~s}$ of PNF followed by 40\% of 1RM (PNF2); (g) $30 \mathrm{~s}$ of PNF followed by $80 \%$ of 1RM (PNF3); (h) $60 \mathrm{~s} \mathrm{of} \mathrm{PNF}$ followed by $80 \%$ of 1RM (PNF4); (i) $40 \%$ of 1RM with no stretching (NS1); and (j) $80 \%$ of 1RM with no stretching (NS2). In both stretching protocols, shoulder girdle muscle exercises were performed. Results: Stretching volume did not seem to significantly decrease the number of repetitions after a bench press session $(p>0.05)$. However, RPE was greater for PNF2 (28.5 $\pm 1.0 ; p=0.016)$ when compared with PNF1 (26.9 \pm 1.9$)$. Conclusion: Stretching volume does not seem to decrease the maximum number of repetitions after a bench press session; however, greater stretching volume appears to increase perception of effort in women when using PNF stretching of longer duration.
\end{abstract}

Keywords: resistance training, flexibility, women, athletic performance, perception

\section{Introduction}

Physical fitness is comprised of different components, such as body composition, as well as cardiorespiratory, neuromotor, and neuromuscular fitness, which positively influence quality of life and health promotion improvement ${ }^{1}$. Because strength and flexibility are physical aspects that compose muscle and neuromotor fitness, their interaction has garnered the interest of several researchers, and have been the topics of different scientific studies ${ }^{2-4}$. Thus, these variables must be incorporated into exercise programs with satisfactory intensity and volume to develop and maintain adequate levels of strength, muscular resistance, lean mass, and range of motion ${ }^{5,6}$. Flexibility can be trained using different stretching methods such as static, ballistic, and proprioceptive neuromuscular facilitation (PNF), and its main functions are to maintain or increase the range of motion, to prevent injury, to delay muscle pain and, if practiced chronically, to improve physical performance ${ }^{7}$. Thus, these different stretching methods are often used as a complementary part of the warm-up routine ${ }^{8-10}$. Accordingly, the American College of Sports Medicine recommends using 30-60 s of stretching to obtain optimal flexibility benefits ${ }^{1}$. Knowing that muscle strength performance can be influenced by many factors, some studies ${ }^{2,11,12}$ have shown that pre-exercise stretching seems to have negative impact on performance, especially if the activity is highly demanding in terms of muscle strength.

In this context, several studies found acute influences of PNF and/or static stretching (SS) on muscle strength ${ }^{2,13,14}$ and number of repetitions ${ }^{2,4,15}$. Other investigations compared the effects of SS on the rating of perceived exertion (RPE) between men and women ${ }^{16,17}$. Because in addition to exhibiting hormonal variations, women have a higher degree of flexibility when compared to men ${ }^{18-20}$, as well as different musculotendinous stiffness, it is important to conduct research in women. However, no studies have been carried out aiming to examine the acute effects of different PNF and SS volumes on the maximum number of repetitions and RPE in women. Thus, since currently there is no research that has investigated the influence of volume of stretching on the maximum number of repetitions and RPE, it is of great importance to understand its influence as this may allow a reduction of time spent in a warm-up session to upper limbs. In addition, it would allow for an understanding of what 
method and stretching volume promotes a greater RPE in each intensity. Therefore, the aim of this study was to examine and compare the acute effects of different volumes (30 and $60 \mathrm{sec}-$ onds) of PNF and static stretching on the maximum number of repetitions with different intensities ( 40 and $80 \%$ of one repetition maximum [1RM]), and RPE in women. The hypothesis of the present study was that when performed for $60 \mathrm{~s}$, PNF and static stretching would cause significant decreases in the maximum number of repetitions (40 and $80 \%$ of $1 \mathrm{RM}$ ) and increased RPE when compared with $30 \mathrm{~s}$.

\section{Methods}

\section{Subjects}

Eleven recreationally trained women between 18 and 35 years of age participated in the study and performed ten experimental protocols in random order on a crossover design. Recreationally trained was considered as having between one to five years of strength training experience ${ }^{21}$. Sample size was calculated using $\mathrm{G}^{*}$ Power $3.1^{22}$. Based on an a priori analysis, we adopted 0.85 power, $\alpha=0.05$, correlation coefficient of 0.5 , nonsphericity correction of 1 and effect size of 0.30 ; therefore, the value of $n=11$ individuals was calculated. To calculate the sample size, the procedures suggested by Beck $^{23}$ were adopted. This a priori analysis of the statistical power was performed to decrease the probability of type II error and to determine the minimum number of participants required for this study. We found that the sample size was sufficient to provide $86.4 \%$ statistical power. The study included women who had experience in resistance training (bench press exercise) for at least one year and a maximum of five years ${ }^{21}$, with weekly frequency of three to five times a week. Subjects who exhibited any type of musculoskeletal injury of the upper limbs and those who responded positively to any of the items in the Physical Activity Readiness Questionnaire/PAR-Q ${ }^{24}$ were excluded from the study. After the risks and benefits of participating in the study were explained, subjects read and signed an informed consent form prepared according to the declaration of Helsinki. The study was approved by the Human Research Ethics Committee of the University (under protocol 101/2011).

\section{Study design}

Participants visited the laboratory for 12 sessions with 48-72 hours between each session. Anthropometry and muscle strength (1RM test and retest) were measured during the first and second visits. From the third to the $12^{\text {th }}$ visit, the individuals were randomly allocated to the following experimental conditions (Figure 1): (a) three 30 -second SS repetitions followed by three sets at $40 \% 1 \mathrm{RM}$ (SS1); (b) three 60 -second SS repetitions followed by three sets at $40 \% 1 \mathrm{RM}(\mathrm{SS} 2)$; (c) three 30 -second $\mathrm{SS}$ repetitions followed by three sets at $80 \% 1 \mathrm{RM}(\mathrm{SS} 3)$; (d) three 60 -second SS repetitions followed by three sets at $80 \% 1 \mathrm{RM}(\mathrm{SS} 4)$; (e) three 30-second PNF stretching repetitions followed by three sets at 40\% 1RM (PNF1); (f) three 60-second PNF stretching repetitions followed by three sets at 40\% 1RM (PNF2); (g) three 30 -second PNF stretching repetitions followed by three sets at $80 \% 1 \mathrm{RM}$ (PNF3); (h) three 60 -second PNF stretching repetitions followed by three sets at $80 \% 1 \mathrm{RM}(\mathrm{PNF} 4)$; (i) three repetitions at 40\% $1 \mathrm{RM}$ with no stretching (NS1); and (j) three sets at $80 \% 1 \mathrm{RM}$ with no stretching (NS2).

Figure 1. Experimental design flowchart.

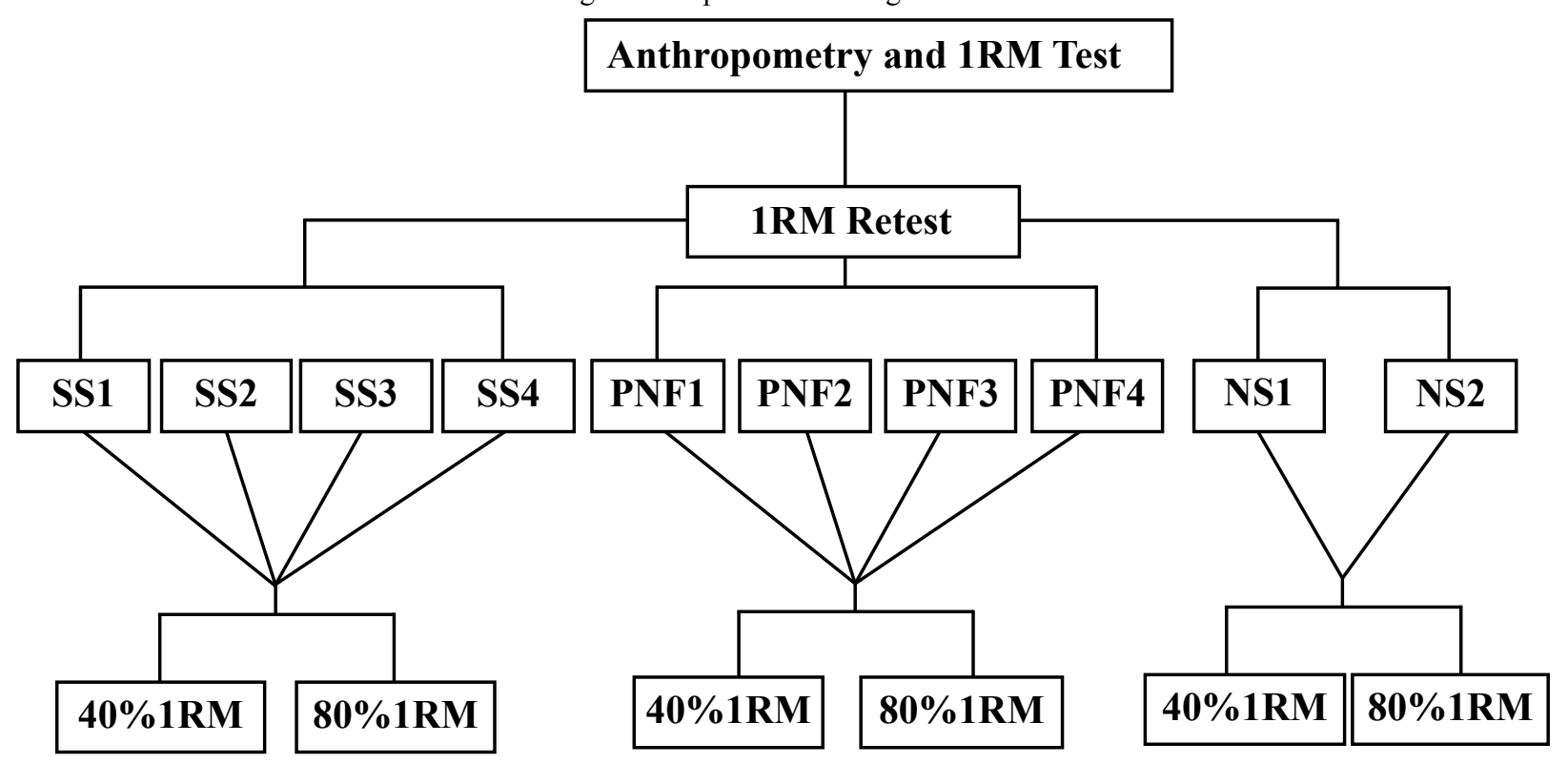

SS1 $=30$ seconds of SS followed by $40 \%$ of 1 RM; SS2 $=60$ seconds of SS followed by $40 \%$ of 1 RM; SS3 $=30$ seconds of SS followed by $80 \%$ of 1 RM; SS4 $=$ 60 seconds of SS followed by $80 \%$ of 1RM; PNF1 $=30$ seconds of PNF stretching followed by $40 \%$ of 1RM; PNF2 $=60$ seconds of PNF stretching followed by $40 \%$ of 1RM; PNF3 $=30$ seconds of PNF stretching followed by $80 \%$ of 1 RM; PNF $4=60$ seconds of PNF stretching followed by $80 \%$ of 1 RM; NS1 $=40 \%$ of 1 RM with no stretching; and NS2 $=80 \%$ of 1 RM with no stretching. 


\section{Procedures}

\section{One Repetition Maximum Test (IRM)}

To obtain load reliability for the bench press exercise 1RM tests, a test and retest were performed on two non-consecutive days $^{25}$. For a warm-up, each individual performed two series of 5 to 10 repetitions with 40 and $60 \%$ of their maximum perceived strength, with 1-minute intervals between sets. After a 1-minute rest period, the third set was performed with 3 to 5 repetitions of 60 to $80 \%$ of the maximum perceived strength. After another period of rest (one minute), the strength assessment was initiated, in which up to five attempts could be performed, adjusting the load before each new attempt. The recovery time between the attempts was standardized to 3 to 5 minutes. The test was stopped when the individual was not able to perform the movement correctly and the maximum load used in the last successful attempt was considered the 1RM.

The distance between the hands corresponded with the position where the humerus was horizontal with the floor and the angle between the arm and forearm was at $90^{\circ}$ in the end of the eccentric phase of the movement. The bench press exercise was performed in the following manner: 1) Initial position - eccentric phase of the movement, initiating from the position of elbow extension; 2) Intermediate position - concentric phase of the movement, with elbows forming na angle of 90 and the humerus parallel with the floor (amplitude limit), returning to the initial position. The following strategies were adopted to decrease the margin of error in data collection procedures: 1) standardized instructions were provided before the tests such that each tested subject was aware of the entire data collection routine; 2) tested individuals were instructed regarding the adequate technique to perform the exercise; 3 ) all participants received standardized verbal encouragement during the tests; and 4) all tests were performed at the same time of day in each session. The highest load achieved in the two days was considered as the 1RM load.

\section{Stretching Protocol}

For SS, three repetitions were performed, keeping the initial position for 30 or 60 seconds in each repetition ${ }^{1,5}$, in which no movement was performed when subjects reached a point of slight discomfort. For the PNF protocol, three repetitions with six seconds of isometric contractions were performed, with two repetitions of six seconds interspersed in the stretched position for the 30 -second volume, and for the 60 -second volume, six repetitions with six seconds of isometric contractions were performed, with four six-second repetitions interspersed in the stretched position ${ }^{1,5}$. For the PNF stretching, isometric muscle actions were performed to the point of slight discomfort. Hence, for each new set of stretching, a new point of discomfort was obtained. These procedures were adopted such that the stretching volume was equal among protocols (SS and PNF), 1.5 mins ( 30 -second volume) or 3 mins ( 60 -second volume). A 30-second interval was used between the stretching repetitions. In both stretching protocols, shoulder girdle muscle exercises were performed. The shoulder girdle was stretched as follows: the volunteer remained seated and performed a horizontal abduction of the glenohumeral joint to the point of slight discomfort. The movement was performed with the elbows flexed to prevent passive insufficiency of the brachial biceps (Figure 2).

Figure 2. Stretching exercise for the shoulder girdle.

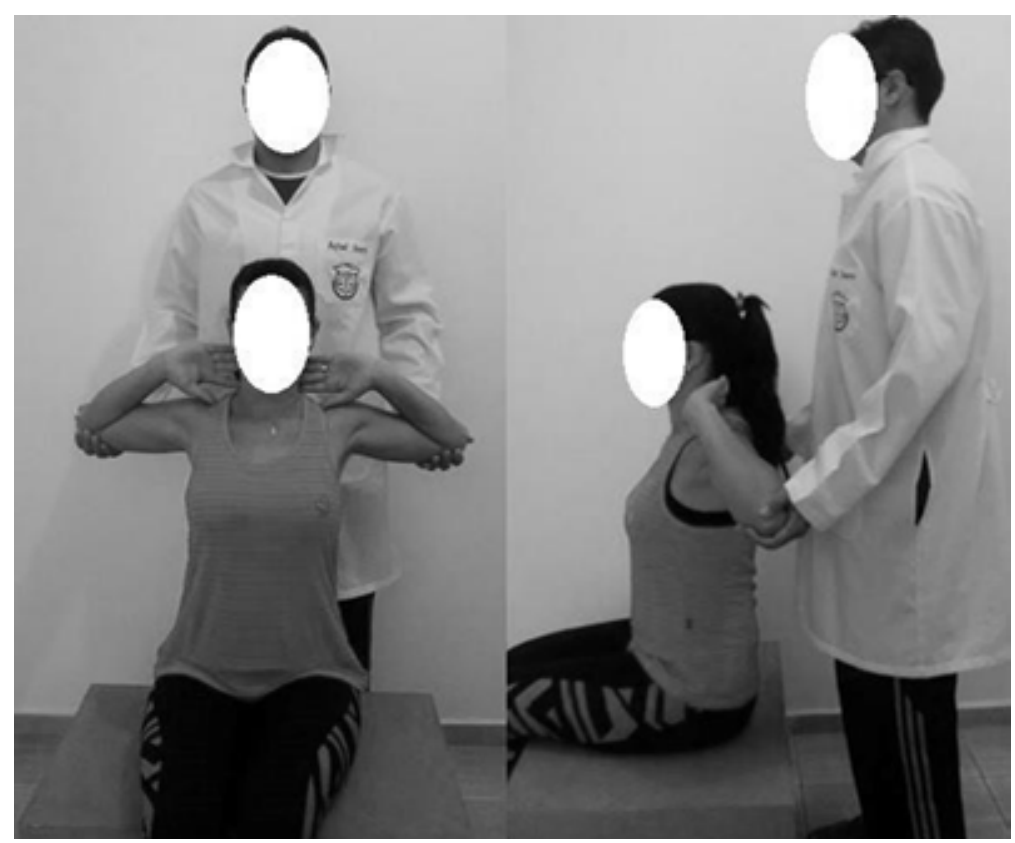




\section{Exercise Procedures}

Strength performance was calculated by adding the number of repetitions performed in three sets of the bench press exercise achieved in a set with fixed load of 40 or $80 \%$ of 1 RM after each protocol, with an interval of one minute between sets. The range of joint movement of elbow in bench press exercise was 90 to $0^{\circ}$, and the speed was controlled by metronome (PHX-209 ${ }^{\circledR}$ - Brazil). The total execution time of the movement was four seconds (two seconds for concentric action and two seconds for eccentric action) until concentric failure occurred. When the individual was not able to maintain the repetition cycle within the set in the cadence and range of motion pre-established, the concentric failure point was determined, and the greatest number of successfully performed repetitions was computed. To control the range of motion, two rods were adapted, and a rubber band was placed between them, which was positioned according to the angle and position of the participant, who, in the eccentric phase $\left(90^{\circ}\right)$, was instructed to touch and perform the concentric phase $\left(0^{\circ}\right)$.

\section{Rating of Perceived Exertion}

Before the start of the study, the participants participated in two familiarization sessions with the OMNI-Resistance Exercise Scale (OMNI-RES) ${ }^{26}$. The RPE was measured after the end of each exercise set (general perception) and the three sets were summed to perform the statistical analysis. This scale was used with a response scale of 0 (extremely easy) to 10 points (extremely difficult). In addition to numerical indications, pictures of effort that represent an individual strength whose posture changes as they increase the scale categories conveying impression of greater effort, were depicted on the scale.

\section{Statistical Analyses}

Data normality was determined using the Shapiro-Wilk test, and the homogeneity of variances was verified using Levene's test. The variables exhibited normal distribution and homogeneity $(p>0.05)$. Test-retest reliability was assessed at percentage of load (1RM) using the intra-class correlation coefficient (ICC), standard error of the measurement (SEM), minimal difference $(\mathrm{MD})^{27}$, and paired samples t-tests. Descriptive data are expressed as means and standard deviations. Two-way repeated measures ANOVA [protocols (SS $30 \mathrm{~s} v s$. SS $60 \mathrm{~s} v s$. PNF $30 \mathrm{~s} v s$. PNF $60 \mathrm{~s} v s . \mathrm{NS}) \times \operatorname{load}(40 \% \mathrm{vs} .80 \%)]$ were used to compare the number of repetitions. When appropriate, Bonferroni post hoc test was used to determine specific differences. Non-parametric Friedman and Wilcoxon tests were used to analyze the RPE. The effect size (ES) was used to verify the magnitudes [trivial $<0.35$, small $=0.35-0.80$, moderate $=0.80-1.50$ and large $>1.5]$ of changes between assessments of the protocols ${ }^{21}$. The significance level was set at $p<0.05$. All statistical analyses were performed using SPSS version 20.0 (SPSS Inc., Chicago, IL).

\section{Results}

The subjects had the following characteristics: $26.45 \pm$ 5.27 years old, $58.77 \pm 6.88 \mathrm{~kg}, 159.27 \pm 7.51 \mathrm{~cm}, 23.11 \pm$ $1.39 \mathrm{~m}^{2} \cdot \mathrm{kg}^{-1}$. The test-retest ICC for the percentage of load was $r=0.941$. The SEM, MD, and $\mathrm{p}$ values from the paired samples t-tests for the test-retest percentage of load were 0.69 , $1.90,0.676$, respectively. The results from each of the paired samples t-tests indicated that there were no mean differences between the percentage of load values from trials 1 and 2 for any of the attempts. The mean and standard deviation of the test and retest of $1 \mathrm{RM}$ were $27.72 \pm 2.86$ and $27.90 \pm 2.87$, respectively.

In the comparative two-way repeated measures ANOVA, there were no significant interactions for protocols $\times$ load $\left(F=1.432 ; \eta^{2}=0.067 ; p=0.231\right)$ and no significant main effect the protocols $\left(F=1.057 ; \eta^{2}=0.050 ; p=0.383\right)$. However, there was a significant main effect for load $\left(F=39.775 ; \eta^{2}=0.665\right.$; $p<0.001)$, as shown in Figure $3 \mathrm{~A}$ and B. The number of repetitions was higher for the $40 \% v s .80 \%$ of $1 \mathrm{RM}$ load for all protocols $(p<0.001)$. In addition, a statistical analysis by sets was performed and no differences were found between the stretching methods $(\mathrm{p}>0.05)$, Table 1 .

Figure 3. Comparative analysis of the number of repetitions of the three series among the protocols.
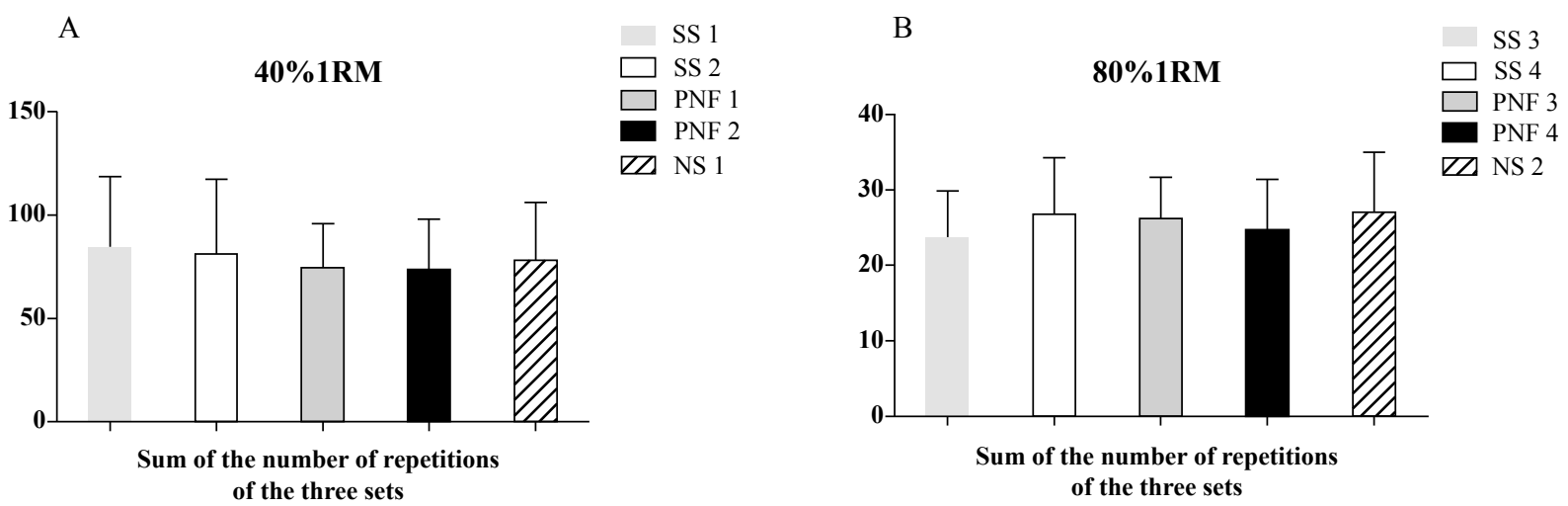

SS1 $=30$ seconds of SS followed by $40 \%$ of 1RM; SS2 $=60$ seconds of SS followed by $40 \%$ of 1 RM; SS $3=30$ seconds of SS followed by $80 \%$ of 1 RM; SS $4=60$ seconds of SS followed by $80 \%$ of 1 RM; PNF $1=30$ seconds of PNF stretching followed by $40 \%$ of 1 RM; PNF $2=60$ seconds of PNF stretching followed by $40 \%$ of 1RM; PNF3 = 30 seconds of PNF stretching followed by $80 \%$ of 1RM; PNF4 $=60$ seconds of PNF stretching followed by $80 \%$ of 1 RM; NS1 $=40 \%$ of 1 RM with no stretching; and NS2 $=80 \%$ of 1 RM with no stretching. 
Table 1. Number of repetitions for each set.

\begin{tabular}{|c|c|c|c|c|}
\hline \multirow[b]{2}{*}{ Stretching } & \multirow[b]{2}{*}{$\%$ of $1 R M$} & \multicolumn{3}{|c|}{ Number of repetitions } \\
\hline & & 1st set & 2nd set & 3rd set \\
\hline \multirow{2}{*}{ SS 30s } & $40 \% 1 \mathrm{RM}$ & $40.9 \pm 22.3$ & $22.6 \pm 8.3$ & $20.8 \pm 8.5$ \\
\hline & $80 \% 1 \mathrm{RM}$ & $9.3 \pm 3.4$ & $7.5 \pm 2.2$ & $6.8 \pm 1.6$ \\
\hline \multirow{2}{*}{ SS 60s } & $40 \% 1 \mathrm{RM}$ & $36.1 \pm 23.6$ & $24.1 \pm 10.2$ & $20.8 \pm 7.9$ \\
\hline & $80 \% 1 \mathrm{RM}$ & $10.4 \pm 4.4$ & $8.7 \pm 1.9$ & $7.5 \pm 1.7$ \\
\hline \multirow{2}{*}{ PNF 30s } & $40 \% 1 \mathrm{RM}$ & $32.8 \pm 15.5$ & $22.3 \pm 7.1$ & $19.3 \pm 6.1$ \\
\hline & $80 \% 1 \mathrm{RM}$ & $10.5 \pm 2.9$ & $8.5 \pm 2.4$ & $7.1 \pm 1.6$ \\
\hline \multirow{2}{*}{ PNF 60s } & $40 \% 1 \mathrm{RM}$ & $31.4 \pm 15.6$ & $22.1 \pm 8.2$ & $19.8 \pm 6.3$ \\
\hline & $80 \% 1 \mathrm{RM}$ & $9.3 \pm 3.2$ & $8,0 \pm 2.1$ & $7.3 \pm 1.6$ \\
\hline \multirow{2}{*}{ NS } & $40 \% 1 \mathrm{RM}$ & $33.0 \pm 19.7$ & $25.2 \pm 8.7$ & $19.7 \pm 6.6$ \\
\hline & $80 \% 1 \mathrm{RM}$ & $10.6 \pm 3.8$ & $9.0 \pm 2.2$ & $7.3 \pm 2.2$ \\
\hline
\end{tabular}

SS = static stretching; PNF = proprioceptive neuromuscular facilitation; NS = no stretching.

The sum of RPE was higher for PNF2 $(28.5 \pm 1.0 ; p=0.016$; $\mathrm{ES}=0.84 /$ magnitude $=$ moderate) when compared with the
PNF1 test $(26.9 \pm 1.9)$ according to the Wilcoxon test, as shown in Figure 4A.

Figure 4. Comparative analysis of the sum of the perception of effort of the three series among the protocols.

A

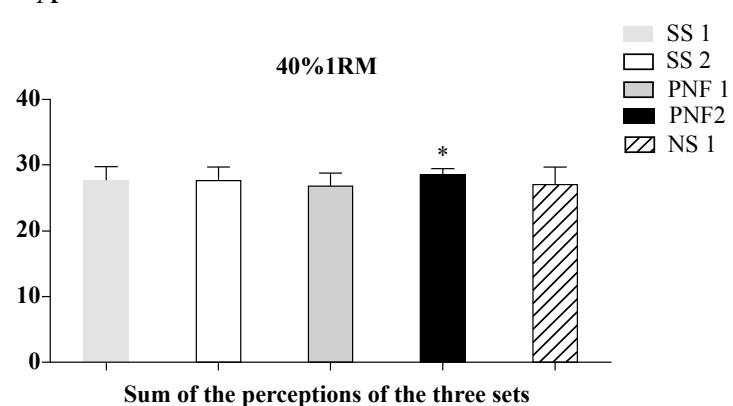

$\mathrm{B}$

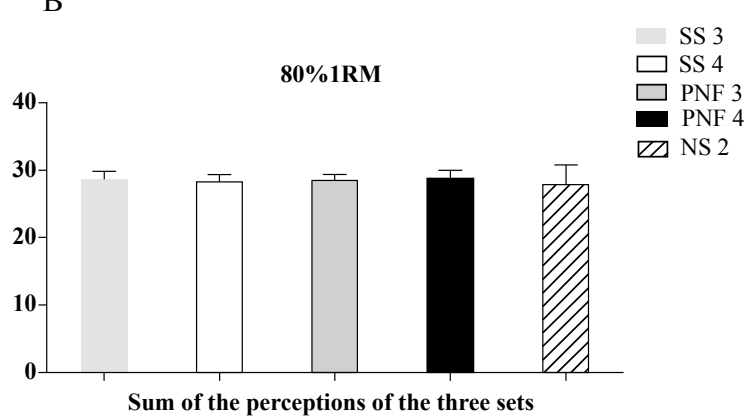

* Significant differences between PNF 2 vs. PNF $1 ;$ SS1 $=30$ seconds of SS followed by $40 \%$ of 1 RM; SS2 $=60$ seconds of SS followed by $40 \%$ of 1 RM; SS $3=$ 30 seconds of SS followed by $80 \%$ of 1RM; SS $4=60$ seconds of SS followed by $80 \%$ of 1RM; PNF1 $=30$ seconds of PNF stretching followed by $40 \%$ of 1 RM; PNF2 $=60$ seconds of PNF stretching followed by $40 \%$ of 1 RM; PNF3 $=30$ seconds of PNF stretching followed by $80 \%$ of 1 RM; PNF $4=60$ seconds of PNF stretching followed by $80 \%$ of 1RM; NS1 $=40 \%$ of 1 RM with no stretching; and NS2 = 80\% of 1 RM with no stretching.

\section{Discussion}

The present study analyzed the acute effects of different stretching volumes (30 and 60 seconds) of the PNF and SS methods on the sum of the number repetitions ( 40 and $80 \%$ of $1 \mathrm{RM})$ and RPE in women. To our knowledge, this study was the first to compare the effects of the stretching volume of the PNF and SS methods on the maximum number of repetitions and RPE. The main result was that stretching volume did not seem to significantly decrease the number of repetitions after a bench press session performed at 40 and $80 \%$ of 1 RM.
However, the greater stretching volume appeared to increase RPE after PNF stretching only in the percentage of $40 \%$. Another interesting finding was that no significant differences were found in the number of repetitions between stretching methods (SS and PNF) when compared with the no stretching control. Although no studies have investigated the effects of stretching volume of the PNF and SS methods on the number of repetitions and RPE in women, some studies have investigated the acute influence of PNF and/or static stretching on strength $^{2,12,14}$ and number of repetitions ${ }^{2,15,28}$ in men. However, only three studies reported an influence of these stretching 
methods on the maximum number of repetitions of the bench press exercise ${ }^{2,12,14}$.

The findings of Franco, Signorelli, Trajano, De Oliveira ${ }^{12}$ and Gomes, Simão, Marques, Costa, Novaes ${ }^{2}$ differ from our findings because those authors only reported significant decreases in the maximum number of repetitions after PNF stretching. This may have occurred because Franco, Signorelli, Trajano, De Oliveira ${ }^{12}$ and Gomes, Simão, Marques, Costa, Novaes ${ }^{2}$ investigated men, whereas present study has assessed women. The differences between studies may be related to sex because in addition to exhibiting hormonal variations ${ }^{29}$ women have a higher degree of flexibility when compared to $\operatorname{men}^{18-20}$, as well as different musculotendinous stiffness. Thus, the decreased maximum number of repetitions reported by Franco, Signorelli, Trajano, De Oliveira ${ }^{12}$ and Gomes, Simão, Marques, Costa, Novaes ${ }^{2}$ may have been due to decreased neural activation induced by the Golgi tendon organ, which is located in the myotendinous junction and is responsible for detecting high force combined with muscle lengthening ${ }^{11}$, a combination that may have been higher in men. Hence, the time under stretch used in the present study may have been too low to promote changes in neural activation in women. This fact may explain the possible similarity in the performance of the number of repetitions between methods with and without stretching. Women are more flexible than $\operatorname{men}^{18,30}$, and the stimuli provided by stretching methods may not have generated enough muscle fatigue and neural activation, which can also explain the non-significant difference in the sum of the number of repetitions between the study protocols (PNF $v s$. SS vs. NS). The study by Silveira, Farias, Alvarez, Bif, Vieira ${ }^{14}$ corroborates our findings as no significant differences were reported in the number of repetitions in the unilateral exercise after 10, 20, and 40 seconds of SS. However, the intensity used for the maximum repetitions test was relatively low $(10 \%$ of $1 \mathrm{RM}$ ) and only one set was performed, which may be a limiting factor of the study (i.e., not exhibiting real characteristics of the intervention practice). Additionally, the stretching volume does not seem to influence the maximum number of repetitions in $\mathrm{men}^{14}$ and women only after SS. The greatest volume used in PNF stretching promoted a higher RPE than the lowest stretching volume. This difference may have occurred because the viscoelastic structures underwent mechanical changes due to the action of strength exercise, which combined with the neural effects caused by the high volume of the PNF stretching method ${ }^{31}$, contributed to the higher RPE of the bench press exercise when compared with the low volume and SS stretching. Moreover, the reduction of neural activation would be one of the reasons for reduced performance. This decrease can be related to the reflection of the Golgi Tendon Organs, nociceptor feedback of pain, and/or fatigue ${ }^{11}$. Thus, it is speculated that the PNF stretching may have reduced the neural activation, which led to increased pain and muscle fatigue, which subsequently caused a higher RPE. According to Alencar and Matias ${ }^{32}$, when the muscle fiber sarcomeres are stretched to a longer length, the overlapping zone decreases, consequently, the tension that can be generated by the fiber is reduced. In addition, it is possible the isometric contractions of PNF during the stretching protocol could have led to an increased RPE. Thus, these mechanisms may have also promoted a greater RPE after SS (knee flexors) compared with no stretching ${ }^{16,17}$.

In conclusion, different SS and PNF volumes do not seem to decrease the number of repetitions during a session of bench press in recreationally trained women. However, the greater stretching volume seems to increase perception of effort after PNF stretching. Because the static and PNF (30 and 60 seconds) stretching volumes did not decrease the number of repetitions performed at 40 and $80 \%$ of $1 \mathrm{RM}$ during a bench press session, it is recommended that strength and conditioning coaches and allied health professionals can work with a higher stretching volume in women, since it did not reduce muscle strength. Future studies should be conducted to examine other stretching volumes with different methods of stretching and in other muscle groups, as well as populations with different resistance training experience.

\section{References}

1. ACSM. American College of Sports Medicine position stand. Quantity and quality of exercise for developing and maintaining cardiorespiratory, musculoskeletal, and neuromotor fitness in apparently healthy adults: guidance for prescribing exercise. Med Sci Sports Exerc 2011;43:1334-59.

2. Gomes TM, Simão R, Marques MC, Costa PB, Novaes JS. Acute effects of two different stretching methods on local muscular endurance performance. J Strength Cond Res 2011;25:745-52.

3. Kay AD, Blazevich AJ. Effect of acute static stretch on maximal muscle performance: a systematic review. Med Sci Sports Exerc 2012;44:154-64.

4. Sá MA, Neto GR, Costa PB, Gomes TM, Bentes CM, Brown $\mathrm{AF}$, et al. Acute effects of different stretching techniques on the number of repetitions in a single lower body resistance training session. J Hum Kinet 2015;45:177-85.

5. ACSM. American College of Sports Medicine position stand. The recommended quantity and quality of exercise for developing and maintaining cardiorespiratory and muscular fitness, and flexibility in healthy adults. Med Sci Sports Exerc 1998;30:975-91.

6. Williams MA, Haskell WL, Ades PA, Amsterdam EA, Bittner V, Franklin BA, et al. Resistance exercise in individuals with and without cardiovascular disease: 2007 update a scientific statement from the american heart association council on clinical cardiology and council on nutrition, physical activity, and metabolism. Circulation 2007;116:572-84.

7. Barroso R, Tricoli V, Gil SS, Ugrinowitsch C, Roschel H. Maximal strength, number of repetitions, and total volume are differently affected by static-, ballistic-, and proprioceptive neuromuscular facilitation stretching. J Strength Cond Res 2012;26:2432-7.

8. Higgs F, Winter SL. The effect of a four-week proprioceptive neuromuscular facilitation stretching program on isokinetic torque production. J Strength Cond Res 2009;23:1442-7.

9. Dallas G, Smirniotou A, Tsiganos G, Tsopani D, Di Cagno A, Tsolakis C. Acute effect of different stretching methods on flexibility and jumping performance in competitive artistic gymnasts. J Sports Med Phys Fitness 2014;54:683-90.

10. Despina T, George D, George T, Sotiris P, George K, Maria R, et al. Short-term effect of whole-body vibration training on balance, 
flexibility and lower limb explosive strength in elite rhythmic gymnasts. Hum Mov Sci 2014;33:149-58.

11. Fowles JR, Sale DG, MacDougall JD. Reduced strength after passive stretch of the human plantarflexors. J Appl Physiol 2000;89:1179-88.

12. Franco BL, Signorelli GR, Trajano GS, De Oliveira CG. Acute effects of different stretching exercises on muscular endurance. J Strength Cond Res 2008;22:1832-7.

13. Nelson AG, Kokkonen J, Arnall DA. Acute muscle stretching inhibits muscle strength endurance performance. J Strength Cond Res 2005;19:338-43.

14. Silveira RN, Farias JM, Alvarez BR, Bif R, Vieira J. Acute effect of static stretching in agonist muscle on the levels of activation and on strength performance of trained men. Rev Bras Med Esporte 2011;17:26-30.

15. Miranda HL, Maia MF, Paz GA, Costa PB. Acute effects of antagonist static stretching in the inter-set rest period on repetition performance and muscle activation. Res Sports Med 2015;23:37-50.

16. Heuser M, Pincivero D. The effects of stretching on knee flexor fatigue and perceived exertion. J Sports Sci 2010;28:219-26.

17. Laur DJ, Anderson T, Geddes G, Crandall A, Pincivero DM. The effects of acute stretching on hamstring muscle fatigue and perceived exertion. J Sports Sci 2003;21:163-70.

18. Carvalho ACG, Paula KC, Azevedo TMC, Nóbrega ACL. Relationship between muscular strength and flexibility in healthy adults of both genders. Rev Bras Med Esporte 1998;4:2-8.

19. Costa PB, Ryan ED, Herda TJ, Walter AA, Hoge KM, Cramer JT. Acute effects of passive stretching on the electromechanical delay and evoked twitch properties: a gender comparison. J Appl Biomech 2012;28:645-54.

20. Hoge KM, Ryan ED, Costa PB, Herda TJ, Walter AA, Stout JR, et al. Gender differences in musculotendinous stiffness and range of motion after an acute bout of stretching. J Strength Cond Res 2010;24:2618-26.

21. Rhea MR. Determining the magnitude of treatment effects in strength training research through the use the effect size. J Strength Cond Res 2004; 18:918-20.

22. Faul F, Erdfelder E, Lang AG, Buchner A. G* Power 3: A flexible statistical power analysis program for the social, behavioral, and biomedical sciences. Behav Res Methods 2007;39:175-91.

23. Beck TW. The importance of a priori sample size estimation in strength and conditioning research. J Strength Cond Res 2013;27:2323-37.
24. Shephard RJ. PAR-Q, Canadian Home Fitness Test and exercise screening alternatives. Sports Med 1988;5:185-95.

25. ACSM. American College of Sports Medicine. ACSM's Guidelines for Exercise Testing and Prescription. 6th ed ed. Baltimore, MD: Williams \& Wilkins; 2000.

26. Robertson RJ, Goss FL, Rutkowaski J, Lenz B, Dixon C, Timmer $\mathrm{J}$, et al. Concurrent validation of the OMNI perceived exertion scale for resistance exercise. Med Sci Sports Exerc 2003;35:333.

27. Weir JP. Quantifying test-retest reliability using the intraclass correlation coefficient and the SEM. J Strength Cond Res 2005;19:231-40.

28. Keese F, Farinatti P, Massaferri R, Matos-Santos L, Silva N, Monteiro W. Acute effect of proprioceptive neuromuscular facilitation stretching on the number of repetitions performed during a multiple set resistance exercise protocol. J Strength Cond Res 2013;27:3028-32.

29. Jonge XAKJ. Effects of the menstrual cycle on exercise performance. Sports Med 2003;33:833-51.

30. Araújo CGS. Flexibility assessment: normative values for flexitest from 5 to 91 years of age. Arq Bras Cardiol 2008;90:280-7.

31. Lieber R. The physiological basis of rehabilitation: skeletal muscle structure, function, and plasticity. $3^{\mathrm{a}}$ ed. Philadelphia: Lippincott Williams \& Wilkins; 2010.

32. Alencar TAM, Matias KFS. Physiological principles of warm-up and muscle stretching on sports activities. Rev Bras Med Esporte 2010;16:230-4.

\section{Corresponding author}

Dr. Gabriel Rodrigues Neto

Coordination of Physical Education , Nursing and Medical Schools, Nova Esperança (FAMENE/ FACENE). Avenida Frei Galvão, 12 - Gramame, PB, 58067-698, João Pessoa, Brazil.

Email: gabrielrodrigues_1988@hotmail.com

Manuscript received on October 26, 2017

Manuscript accepted on May 28, 2018

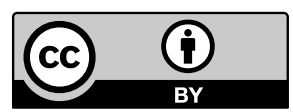

Motriz. The Journal of Physical Education. UNESP. Rio Claro, SP, Brazil - eISSN: 1980-6574 - under a license Creative Commons - Version 4.0 